\title{
Realistic Rendering of Birefringency in Uniaxial Crystals
}

\author{
ANDREA WEIDLICH and ALEXANDER WILKIE \\ Institute of Computer Graphics and Algorithms, Vienna University of Technology
}

\begin{abstract}
In this paper we derive the complete set of formulas which is needed to generate physically plausible images of uniaxial crystals. So far no computer graphics publication contains all the formulas one needs to compute the interaction of light with such crystals in a form that is useable by a graphics application, especially if a polarisation-aware rendering system is being used.

This paper contains the complete derivation of the Fresnel coefficients for birefringent transparent materials, as well as for the direction cosines of the extraordinary ray and the Mueller matrices necessary to describe polarisation effects. The formulas we derive can be directly used in a ray based renderer, and we demonstrate these capabilities in test scenes.
\end{abstract}

Categories and Subject Descriptors: I.3.7 [Computer Graphics]: Three-Dimensional Graphics and Realism

General Terms: Theory, Algorithms

Additional Key Words and Phrases: Birefringence, crystals, polarisation

\section{INTRODUCTION}

Crystals can exhibit a number of interesting optical properties, such as double refraction, optical rotation or polarisation effects. So far, the rendering community has only made a few isolated attempts to simulate these phenomena.

The reasons for the apparent reluctance to investigate these effects are twofold: most of them do not cause very prominent changes in object appearance, and - in contrast to "normal" refraction and reflection from isotropic transparent objects, which is easy to understand - the theoretical foundation needed for getting them right is not trivial.

Since most of the time computer graphics is about generating believable images (as opposed to radiometrically exact ones) the fact that no complete, immediately reproducible treatment of some of these effects has been published so far has not been perceived as a significant omission up to now.

However, in the gemstone industry, computer-aided prototyping of gemstone cuts is slowly becoming a standard; as a consequence, the computation of physically accurate images of crystals is increasing in importance. But practically all of the rendering solutions which have been developed for this area so far assume the gemstones to be plain isotropic materials; this is an assumption which can cause inaccuracies and mispredictions for those types of crystals where one of the neglected effects - such as birefringence - is a prominent feature.

The aim of this paper is to complete the computer graphics knowledge of crystal rendering in one particular problem area, and to derive all the formulas one needs to implement a ray-based renderer for uniaxial, birefringent crystals.

Author's address: Andrea Weidlich, Institut fuer Computergraphik und Algorithmen, Technische Universitaet Wien, Favoritenstrasse 9-11/186, A-1040 Wien, Austria.

Permission to make digital/hard copy of all or part of this material without fee for personal or classroom use provided that the copies are not made or distributed for profit or commercial advantage, the ACM copyright/server notice, the title of the publication, and its date appear, and notice is given that copying is by permission of the ACM, Inc. To copy otherwise, to republish, to post on servers, or to redistribute to lists requires prior specific permission and/or a fee.

(c) 20TBD ACM 0730-0301/20TBD/0100-0111 $\$ 5.00$

ACM Transactions on Graphics, Vol. TBD, No. TBD, TBD 20TBD, Pages 111-0??. 


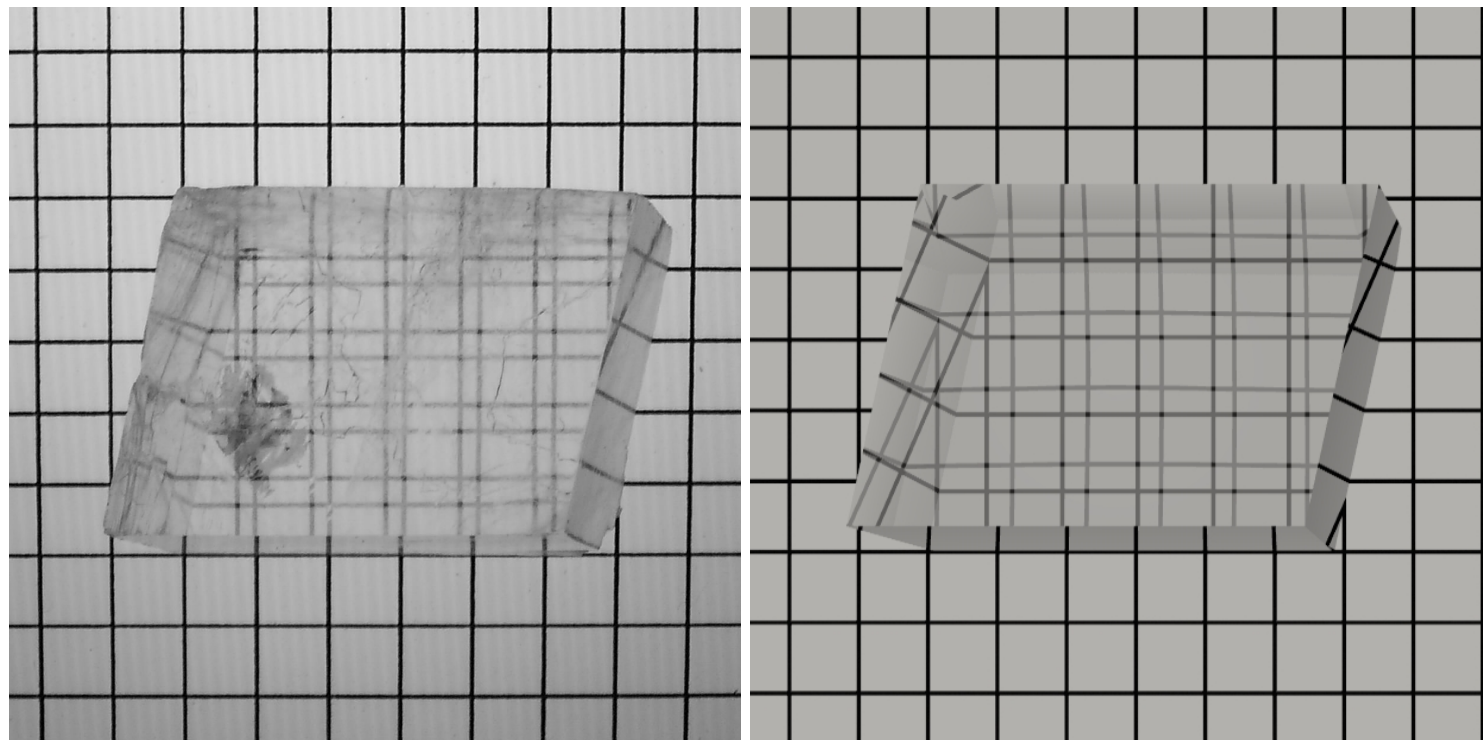

Fig. 1. A photograph (left) and rendered image (right) of a calcite crystal placed on a luminescent background with a grid pattern. The setting was chosen in order to maximise the visibility of the birefringence effects, and the formulas derived in this paper were used for calculation of the synthetic image. The remaining differences in the refraction patterns can be traced to small differences in the camera parameters as well as volume inhomogeneities and surface irregularities in the real sample calcite.

\subsection{Structure of this Paper}

We first discuss the state of the art in crystal rendering in section 2, give a short general overview of the physical background needed to understand optical properties of uniaxial crystals in section 3 , and then identify the distinguishing aspects of the problem of ray propagation in such crystals in section 3.4.

In sections 3, 4, 5 and 6 we then cover all these sub-problems in turn by deriving the formulas needed for an implementation, i.e. the refraction index for the extraordinary ray, the Fresnel coefficients, the direction cosines of the e-ray and the Mueller matrix which is needed in polarisation-aware rendering systems.

The resulting formulas which are to be used in a renderer are spread through sections $3,4,5$ and 6 . Therefore we give a concise overview of what exactly is to be done if one wants to perform raycasting operations on a birefringent interface in section 7 .

We conclude by demonstrating the viability of this approach by testing it in various representative settings in section 8 , and giving some directions for future work in section 9 .

\section{RELATED WORK}

Light which passes through a crystal can be subjected to a wide variety of optical effects; some are peculiar to crystals, while others can be found in all transparent materials. Practically all of them have already been simulated in a computer graphics system at some time, although some of these efforts did not yield results that are immediately useable in the context of a modern rendering system, or were inefficient proof-of-concept studies.

With the notable exception of [Guy and Soler 2004], who presented an integrated real-time system capable of interactively rendering gemstones including dispersion effects, all the publications discussed in this section deal with ray-based, non-interactive rendering systems. 
Dispersive refraction is perhaps the simplest, but also one of the most visually prominent attributes of all transparent materials. The simulation of this effect was first introduced to computer graphics by [Thomas 1986] as an add-on to a standard ray tracer. Dispersion was obtained by breaking up the incident energy into six subrays, where each one represented only a part of the spectrum, and the incident ray is adaptively subdivided further so that the spread of each refracted ray is small enough. [Yuan et al. 1988] managed to reduce the computational complexity by using only three rays to represent a spreading incident ray.

[Yokoi et al. 1986] published an algorithm to render asterism or chatoyancy effects of gems, and achieved light dispersion through a microfacet model. Dispersion was further investigated by [Sun et al. 2000b], who used a hybrid spectral model which decomposed the spectrum into its smooth component, and a collection of spikes to optimally represent monochromatic light rays and spiky emission spectra.

The exact simulation of light absorption in transparent media is a problem which is frequently underestimated, especially for uni- and biaxial crystals; for these no computer graphics treatment exists so far, and the relevant discussions in crystallographic texts are highly involved.

For normal isotropic materials solutions have been known for a long time, however, and a recent discussion can be found in [Sun et al. 2000a], where they combined the effects of light dispersion with volume absorption to simulate the colour of diamonds. Absorption in homogeneous transparent materials was also investigated in [Sun et al. 1999].

Another prominent feature is the darkening of certain facets in crystals due to polarisation. Among the few publications which discuss polarisation in ray tracing are [Wolff and Kurlander 1990], who were among the first to investigate this problem, and used $2 \times 2$ coherency matrices to represent the polarisation state of light. [Wilkie et al. 2001] used Mueller matrices to avoid the complex numbers of coherency matrices. With this description of polarisation state, fluorescence effects could be included in a natural fashion in a ray tracing system. However, neither of them explicitly dealt with polarisation in the context of crystal optics.

The only authors to discuss ray-based rendering of birefringency were [Tannenbaum et al. 1994], who investigated the behaviour of light-material interactions with optically anisotropic media. They extended the work of Wolff and Kurlander by taking the coherency alterations that occur when a light ray interacts with an anisotropic medium into account, and derived some formulas for the intensity and the direction of the extraordinary ray. However, even in the longer version of the paper - which provides some of the intermediate steps that are missing in the short version found in the SIGGRAPH proceedings - the derivations are difficult to understand, because some crucial steps and end results are still omitted, and those formulas which are provided as end results would be very hard to implement, even if one had a rendering system that can work with coherency matrices.

\section{OPTICAL PROPERTIES OF UNIAXIAL CRYSTALS}

\subsection{Dielectric Tensor}

Computer graphics texts usually assume that materials are isotropic, which means that the propagation characteristics of an electromagnetic wave are independent of its propagation direction within the medium. In such isotropic materials the electric field vector $\mathbf{E}$ is parallel to the dielectric displacement vector $\mathbf{D}$. They are related to each other by the product of the relative permittivity $\epsilon_{r}$ and the permittivity of free space $\epsilon_{0}$, so that $\epsilon=\epsilon_{0} \epsilon_{r}$.

$$
\mathbf{D}=\epsilon \mathbf{E}
$$

In optically anisotropic materials - which include most crystals - the propagation characteristics of a ray depend on the relative orientation of the electric field vector to the inherent axial directions of the crystal. As a consequence $\mathbf{D}$ will no longer necessarily be oriented in the direction of $\mathbf{E}$, and therefore $\epsilon$ has to be in contrast to isotropic transparent material, where the relationship between $\mathbf{E}$ and $\mathbf{D}$ can be described by 
a single scalar - a tensor of rank 2 [Born and Wolf 1999].

$$
\begin{aligned}
& D_{x}=\epsilon_{x x} E_{x}+\epsilon_{x y} E_{y}+\epsilon_{x z} E_{z} \\
& D_{y}=\epsilon_{y x} E_{x}+\epsilon_{y y} E_{y}+\epsilon_{y z} E_{z} \\
& D_{z}=\epsilon_{z x} E_{x}+\epsilon_{z y} E_{y}+\epsilon_{z z} E_{z}
\end{aligned}
$$

The dielectric tensor $\epsilon$ is symmetrical, and hence has only six instead of nine independent components. As a consequence, a coordinate system can be found for any crystal in which the nondiagonal elements in this matrix disappear. By defining such a coordinate system, i.e. finding a rotation that transforms the symmetric tensor into the diagonal form, the components of the dielectric tensor can be reduced to three scalars $\epsilon_{x x}^{\prime}=n_{x}^{2}, \epsilon_{y y}^{\prime}=n_{y}^{2}$ and $\epsilon_{z z}^{\prime}=n_{z}^{2}$. These remaining elements are called the principal dielectric constants, or principal permittivities. The axes of this coordinate system are known as principal axes. With this choice of axes, $\mathbf{D}$ can be written as

$$
\left(\begin{array}{c}
D_{x} \\
D_{y} \\
D_{z}
\end{array}\right)=\left(\begin{array}{ccc}
\epsilon_{x x}^{\prime} & 0 & 0 \\
0 & \epsilon_{y y}^{\prime} & 0 \\
0 & 0 & \epsilon_{z z}^{\prime}
\end{array}\right)\left(\begin{array}{c}
E_{x} \\
E_{y} \\
E_{z}
\end{array}\right) .
$$

\subsection{Types of Crystal Structures}

It is a defining property of crystals to exhibit a periodical arrangement of atoms in an ordered geometrical pattern, which repeats in all three dimensions on a lattice. The exact arrangement is characteristic for each type of crystal, and is referred to as its crystal structure. Seven distinct basic configurations of such structures can be distinguished, and are known as crystal systems. These can be further grouped into three classes according to the relationship between their principal permittivities:

- Crystals with a cubic structure - e.g. Diamonds - are isotropic, and have three equal principal permittivities, $\epsilon_{x x}^{\prime}=\epsilon_{y y}^{\prime}=\epsilon_{z z}^{\prime}$. Snell's law ${ }^{1}$ - the classical law of refraction - can be used to determine the propagation angle for the single refracted ray which occurs in such materials, the direction of which is obviously independent of the relative orientation of incident ray and crystal lattice.

- Crystals with trigonal, tetragonal or hexagonal structures are called uniaxial, and have two equal principal permittivities, $\epsilon_{x x}^{\prime}=\epsilon_{y y}^{\prime} \neq \epsilon_{z z}^{\prime}$. Zirconia and Quartz are examples of uniaxial materials. In such crystals, the refracted part of any incident light wave is split into two wavetrains, which are linearly polarised in orthogonal directions. The first one - the so-called ordinary ray - is polarised perpendicular to the principal plane, obeys Snell's law, and propagates in the same direction as if the material were isotropic; this ray is sometimes also called s-polarised. The second ray - the extraordinary ray - is polarised parallel to the principal plane, and is called $p$-polarised ${ }^{2}$; see figure 2 for an illustration. As their name implies, uniaxial crystals have one optical axis - the one direction within the crystal where ordinary and extraordinary refraction rays coincide.

- Finally, crystals with orthorhombic, monoclinic or triclinic structure are referred to as biaxial materials, as they have two optical axes. Olivine and Kyanite belong to this group. For them, all principal permittivities $\epsilon_{x x}^{\prime} \neq \epsilon_{y y}^{\prime} \neq \epsilon_{z z}^{\prime}$ are different. In biaxial crystals the refracted light wave is split into two extraordinary rays - there is no ordinary ray, which means that no part of the refracted light obeys Snell's law.

\footnotetext{
${ }^{1}$ Snell's law $\left(\sin \theta_{1} \cdot n_{1}=\sin \theta_{2} \cdot n_{2}\right)$ gives the relationship between angle of incidence $\sin \theta_{1}$ and angle of refraction sin $\theta_{2}$ of a light wave when travelling between two media with different refraction indices $n_{1}$ and $n_{2}$.

${ }^{2}$ The terms "s-polarised" and "p-polarised" are derived from the German word "senkrecht" (i.e. perpendicular) and "parallel", respectively, which refer to the oscillation direction of the electric field. The continuing usage of these terms in English literature is apparently a legacy from the days when most optical literature was published in German.
} 

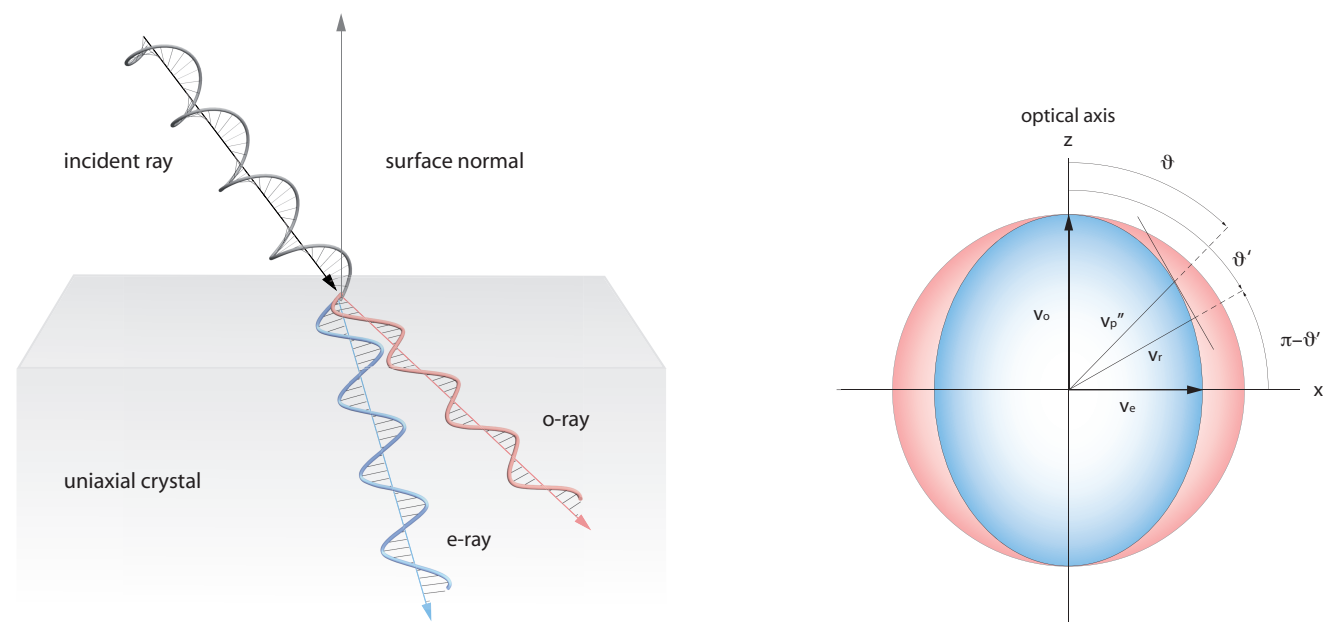

Fig. 2. Left: Split of an incident ray into an ordinary and an extraordinary ray upon incidence on a uniaxial crystal. Right: Ray surface of a positive uniaxial crystal. The ray-velocity surface describes the velocity of rays in their propagation direction, which for e-rays depends on the direction of the wave vectors except for propagation along a principal axis. The semi-axes of the ellipse define the directions of the two permitted polarisation directions which can propagate through the crystal, and the lengths of these semi-axes give the refractive indices experienced by these two polarisations. The surface for o-waves is a sphere, since the refractive index is independent of the propagation direction. Note that the ray velocity $v_{r}=v_{p} \cos \left(\vartheta^{\prime}-\vartheta\right)$.

\subsection{Light Propagation in Uniaxial Crystals}

The refractive index of the extraordinary ray can be derived from Fresnel's equation of wave normals. This equation is biquadratic and has two solutions. For uniaxial crystals with $n_{x}=n_{y} \neq n_{z}$ the two solutions correspond to the ordinary refractive index $n^{\prime}$ and the extraordinary $n^{\prime \prime}$ [Yariv and Yeh 1984]

$$
\begin{aligned}
n^{\prime} & =n_{s}=n_{o} \\
n^{\prime \prime} & =n_{p}=\frac{n_{o} n_{e}}{\sqrt{n_{o}^{2} \sin ^{2} \vartheta^{\prime}+n_{e}^{2} \cos ^{2} \vartheta^{\prime}}}
\end{aligned}
$$

where $\vartheta^{\prime}$ is the angle between the unit wave-normal $\mathbf{s}$ and the $z$-axis. In the case of the electric field being oriented along the crystal coordinate axes, the refractive index $n^{\prime \prime}$ assumes the values $n_{o}$ or $n_{e}$. When the electric field vector points in other directions, $n^{\prime \prime}$ takes on values which are between $n_{o}$ and $n_{e}$.

Evidently, s- and p-polarised light refracts into an anisotropic crystal at two different angles, and has different velocities in the crystal.

- For ordinary rays light behaves the same way regardless of the incident angle, because the refractive index $n$ is independent of $\vartheta^{\prime}$. Therefore, for the ordinary wave the velocity is equal in every direction, and it propagates with wave-normal (or phase) velocity $v_{p}=c_{0} / n$ along $\mathbf{s}$.

- For extraordinary rays the situation is more complicated, since the extraordinary index of refraction exhibits an angular dependence. In this case we have to distinguish between the ray vector (the direction of energy propagation) and the wave-front normal, because, unlike ordinary waves, ray velocity and wavenormal velocity are different. However, the difference is small even for strongly birefringent materials.

$$
\begin{aligned}
v_{p}^{\prime 2} & =v_{o}^{2} \\
v_{p}^{\prime \prime 2} & =v_{o}^{2} \cos ^{2} \vartheta^{\prime}+v_{e}^{2} \sin ^{2} \vartheta^{\prime} .
\end{aligned}
$$


If the ordinary ray is faster than the extraordinary $\left(v_{e}<v_{o}\right)$ a crystal is said to be positive uniaxial. Otherwise, if the ordinary ray is slower than the extraordinary $\left(v_{e}>v_{o}\right)$, the crystal is negative uniaxial. Since the propagation speed is equal in every direction for the ordinary ray, its ray surface is a sphere of radius $v_{p}=v_{o}$. For the extraordinary ray the ray surface is an ellipsoid (figure 2 ).

Usually a part of light is absorbed inside a transparent material by traveling through it; since this is a completely different - and very complex - problem in its own right for crystals, we do not deal with absorption in this paper. For a detailed discussion of the theory of absorption in crystals we e.g. refer the reader to [Szivessy 1928] or [Ramachendran and Ramaseshan 1961]. However, since a large number of minerals exhibit absorption to some degree, it is desirable to have at least an approximate solution. A practicable approach of this type is the solution of [Guy and Soler 2004], who use characteristic constants (e.g. from measured absorption spectra) for both the absorption of ordinary and the extraordinary rays.

\subsection{Calculation of Light Propagation in Uniaxial Crystals}

Four distinct problems have to be solved in order to perform rendering computations which involve the propagation of light in uniaxial materials. These are addressed as follows in the subsequent sections:

(1) The index of refraction which governs the behaviour of the extraordinary ray is not constant - it depends on the incident angle, and the orientation of the crystal axis. The appropriate formulas were already discussed in section 3.3. Note that these formulas are common knowledge and can be found in numerous physics books.

(2) The relative distribution of the refracted light between the $o-$ and $e$-ray has to be determined. This problem is discussed in section 4, where the Fresnel coefficients for an isotropic-uniaxial interface are derived.

(3) Since it usually leaves the plane of incidence, the extraordinary ray clearly does not obey Snell's law, and a different approach is needed to determine its direction. We provide the necessary formulas in section 5 , where the direction cosine of the extraordinary ray is discussed.

(4) If a polarisation-aware rendering system is used, the polarisation state of the two rays has to be determined. The derivation for the Mueller matrices needed in this case is given in section 6.2.

\section{FRESNEL COEFFICIENTS FOR UNIAXIAL CRYSTALS}

The purpose of this section is to formulate the physical properties of crystals in tensor notation and give explicit formulas for the Fresnel coefficients. The Fresnel coefficients describe the behaviour of reflection and refraction and calculate the amount of reflected light relative to the amount transmitted through an interface. The exact values depend on the incident angle, the polarisation and the wavelength of an incident light ray.

Only a few publications on the derivation of Fresnel coefficients for anisotropic materials exist, all of them outside computer graphics. [Lin and $\mathrm{Wu}$ 1998] examined the reflection coefficient for waves which propagate from an isotropic medium to an anisotropic medium and [Schwelb 1987] derived the Fresnel coefficients in terms of the characteristic admittance matrix for anisotropic media which exhibit loss or gain. Other methods deal with a $4 \times 4$ matrix formalism, like the technique proposed by [McClain et al. 1993a], who derived the Fresnel coefficients for anisotropic optically active materials, non-optically active uniaxial materials and isotropic optically active materials. These formulas were used by Guy and Soler in their paper, who then outlined a rendering solution.

The disadvantage of these formalisms is that the eigenvalue problem is not solved; this has to be done by the user. In this paper all calculations are carried through, so that the resulting formulas can be used in a renderer. 
The sequence of derivation we use - which is based on [Lekner 1991] - is the following: we transform the dielectric tensor so that it is given in terms of the dielectric constants and use the result to solve equation (1). Then we can express the electric fields in terms of the optical axis, the refractive indices and the incident ray, and derive the Fresnel coefficients from the boundary conditions.

\subsection{Transformation of the Dielectric Tensor}

As mentioned earlier the dielectric tensor can always be diagonalised. This is an eigenvalue problem which can be solved by rotating the tensor around the $x^{-}, y$ - and $z$-axes of its coordinate system. One way to express the rotation matrix needed for this is to make use of Euler angles. Another possibility is to do an orthogonal transformation, which will be done here. A detailed explanation can be found in [Goldstein 2002].

Any three dimensional vector can be defined by its direction cosines which can be expressed in terms of two sets of Cartesian coordinates. For example, the $\mathbf{x}$-axis is given by its three direction cosines with respect to another set of axes $\mathbf{x}^{\prime}, \mathbf{y}^{\prime}$ and $\mathbf{z}^{\prime}$, so that

$$
\begin{aligned}
& \alpha_{1}=\cos \left(\mathbf{x}^{\prime}, \mathbf{x}\right)=\mathbf{x}^{\prime} \cdot \mathbf{x} \\
& \alpha_{2}=\cos \left(\mathbf{x}^{\prime}, \mathbf{y}\right)=\mathbf{x}^{\prime} \cdot \mathbf{y} \\
& \alpha_{3}=\cos \left(\mathbf{x}^{\prime}, \mathbf{z}\right)=\mathbf{x}^{\prime} \cdot \mathbf{z} .
\end{aligned}
$$

Similar equations can be set up for the direction cosines of the $\mathbf{y}^{\prime}$-axis and the $\mathbf{z}^{\prime}$-axis. Thus the vectors $\mathbf{x}^{\prime}, \mathbf{y}^{\prime}$ and $\mathbf{z}^{\prime}$ can be written as

$$
\begin{aligned}
& \mathbf{x}^{\prime}=\alpha_{1} \mathbf{x}+\alpha_{2} \mathbf{y}+\alpha_{3} \mathbf{z} \\
& \mathbf{y}^{\prime}=\beta_{1} \mathbf{x}+\beta_{2} \mathbf{y}+\beta_{3} \mathbf{z} \\
& \mathbf{z}^{\prime}=\gamma_{1} \mathbf{x}+\gamma_{2} \mathbf{y}+\gamma_{3} \mathbf{z}
\end{aligned}
$$

and the inverse transformation is

$$
\begin{aligned}
& \mathbf{x}=\alpha_{1} \mathbf{x}^{\prime}+\beta_{1} \mathbf{y}^{\prime}+\gamma_{1} \mathbf{z}^{\prime} \\
& \mathbf{y}=\alpha_{2} \mathbf{x}^{\prime}+\beta_{2} \mathbf{y}^{\prime}+\gamma_{2} \mathbf{z}^{\prime} \\
& \mathbf{z}=\alpha_{3} \mathbf{x}^{\prime}+\beta_{3} \mathbf{y}^{\prime}+\gamma_{3} \mathbf{z}^{\prime} .
\end{aligned}
$$

The direction cosines are related to each other, because the axes of both coordinate systems are mutually orthogonal. These relations are called orthogonality relations. Therefore

$$
\mathbf{x} \cdot \mathbf{y}=\mathbf{y} \cdot \mathbf{z}=\mathbf{z} \cdot \mathbf{x}=0
$$

and

$$
\mathbf{x} \cdot \mathbf{x}=\mathbf{y} \cdot \mathbf{y}=\mathbf{z} \cdot \mathbf{z}=1 .
$$

This also holds true between the direction cosines. We now return to the dielectric tensor, which is given by

$$
\epsilon=\left(\begin{array}{ccc}
\epsilon_{x x} & \epsilon_{x y} & \epsilon_{x z} \\
\epsilon_{y x} & \epsilon_{y y} & \epsilon_{y z} \\
\epsilon_{z x} & \epsilon_{z y} & \epsilon_{z z}
\end{array}\right) .
$$

Its components are given with reference to a coordinate system $x, y, z$. Since the dielectric tensor describes a physical quantity, its components transform according to a rule that makes it possible to find the components for any given set of axes. For a second-rank tensor $T$ the transformation law is [Nye 2003]

$$
T_{i j}^{\prime}=\sum_{k} \sum_{l} a_{i k} a_{j l} T_{k l}
$$

ACM Transactions on Graphics, Vol. TBD, No. TBD, TBD 20 TBD. 
where $a_{i j}$ are the elements of the transformation matrix ${ }^{3}$. If we want to transform the dielectric tensor according to this law so that it is given in terms of the principal axes, we have to do an orthogonal transformation. The rotations are represented by the matrix $R$. The transformation law of the tensor can also be formulated by writing the $a_{i k}$-term after the tensor and transposing the result. The transformation of axes for the components of the dielectric tensor is equivalent to

$$
\epsilon=R \cdot \epsilon^{\prime} \cdot R^{-1}=\left(\begin{array}{ccc}
\alpha_{1} & \alpha_{2} & \alpha_{3} \\
\beta_{1} & \beta_{2} & \beta_{3} \\
\gamma_{1} & \gamma_{2} & \gamma_{3}
\end{array}\right) \cdot\left(\begin{array}{ccc}
\epsilon_{x x}^{\prime} & 0 & 0 \\
0 & \epsilon_{y y}^{\prime} & 0 \\
0 & 0 & \epsilon_{z z}^{\prime}
\end{array}\right) \cdot\left(\begin{array}{lll}
\alpha_{1} & \beta_{1} & \gamma_{1} \\
\alpha_{2} & \beta_{2} & \gamma_{2} \\
\alpha_{3} & \beta_{3} & \gamma_{3}
\end{array}\right)
$$

Since we are only interested in the uniaxial case here, we can assume $\epsilon_{x x}^{\prime}=\epsilon_{y y}^{\prime}=\epsilon_{o}$ and $\epsilon_{z z}^{\prime}=\epsilon_{e}$. If we take advantage of the orthogonality relations of the direction cosines, the dielectric tensor reduces to [Lekner 1991]

$$
\epsilon=\left(\begin{array}{ccc}
\alpha_{3}^{2}\left(\epsilon_{e}-\epsilon_{o}\right)+\epsilon_{o} & \alpha_{3} \beta_{3}\left(\epsilon_{e}-\epsilon_{o}\right) & \alpha_{3} \gamma_{3}\left(\epsilon_{e}-\epsilon_{o}\right) \\
\alpha_{3} \beta_{3}\left(\epsilon_{e}-\epsilon_{o}\right) & \beta_{3}^{2}\left(\epsilon_{e}-\epsilon_{o}\right)+\epsilon_{o} & \beta_{3} \gamma_{3}\left(\epsilon_{e}-\epsilon_{o}\right) \\
\alpha_{3} \gamma_{3}\left(\epsilon_{e}-\epsilon_{o}\right) & \beta_{3} \gamma_{3}\left(\epsilon_{e}-\epsilon_{o}\right) & \gamma_{3}^{2}\left(\epsilon_{e}-\epsilon_{o}\right)+\epsilon_{o}
\end{array}\right) .
$$

The direction cosines $\alpha_{3}, \beta_{3}$ and $\gamma_{3}$ coincide with the optical axis.

\subsection{Normal Modes in Uniaxial Crystals}

Light can be seen as an electromagnetic wave and can therefore be thought of as composed of an electric field and a magnetic field. The electric field vector $\mathbf{E}$ and the magnetic field vector $\mathbf{H}$ as well as the magnetic induction $\mathbf{B}$ oscillate perpendicular to each other and to the propagation direction $\mathbf{k}$ as well. Maxwell's equations connect the five quantities $\mathbf{E}, \mathbf{H}, \mathbf{B}$, the dielectric displacement $\mathbf{D}$ and the electric current density j (see [Born and Wolf 1999] for details). Starting from these equations, we now can derive the Fresnel coefficiets for the uniaxial case. To derive these results, we make use of Faraday's law to relate $\mathbf{E}$ and $\mathbf{B}$. The two curl Maxwell equations are then

$$
\nabla \times \mathbf{E}=i k \mathbf{B}
$$

and

$$
\nabla \times \mathbf{B}=-i k \mathbf{D}
$$

with $k=\omega / c=2 \pi / \lambda$ where $\lambda$ denotes the wavelength. $\mathbf{E}$ and $\mathbf{D}$ are related through the dielectric tensor so that after eliminating $\mathbf{B}$ and specialising the resulting equations for the case of anisotropic crystals, three equations remain [Lekner 1991]:

$$
\begin{aligned}
-q^{2} E_{x}+q K E_{z}+k^{2} D_{x} & =0 \\
-\left(q^{2}+K\right) E_{y}+k^{2} D_{y} & =0 \\
-q K E_{x}-K^{2} E_{z}+k^{2} D_{z} & =0 .
\end{aligned}
$$

$q$ is the component of the wave-normal vector and $K$ is the tangential component of all wave vectors, i.e. $K=k n \sin \theta_{i}$. This is equivalent to

$$
\left(\begin{array}{ccc}
\epsilon_{x x}-q^{2} / k^{2} & \epsilon_{x y} & \epsilon_{x z}+q K / k^{2} \\
\epsilon_{y x} & \epsilon_{y y}-\left(q^{2}+K^{2}\right) / k^{2} & \epsilon_{y z} \\
\epsilon_{z x}-q K / k^{2} & \epsilon_{z y} & \epsilon_{z z}-K^{2} / k^{2}
\end{array}\right) \cdot\left(\begin{array}{c}
E_{x} \\
E_{y} \\
E_{z}
\end{array}\right)=0
$$

\footnotetext{
${ }^{3}$ Although both the transformation matrix and the dielectric tensor are arrays of nine numbers, there are some fundamental differences between the two. The coefficients of $a_{i j}$ put two sets of axes into relation, while the components of the tensor represent a physical quantity for one given set of axes.
} 
if we fall back on the dielectric tensor in terms of the dielectric constants and substitute its elements into the tensor matrix. Now we have to find a solution for $\mathbf{E}$. The determinant of the tensor matrix must equal zero. Otherwise the only solution for the electric field is $\mathbf{E}=0$. We get solutions for the ordinary electric field $\mathbf{E}_{o}$ if we solve this eigenvalue problem by replacing $q$ with

$$
q_{o}=\sqrt{\epsilon_{o} k^{2}-K^{2}} .
$$

It is convenient to set $k=\omega / c=1$ [Lekner 1991] and if we also assume $n_{i}=1, E_{o}$ is given in normalized form by

$$
\begin{aligned}
E_{o x} & =\beta \sqrt{-\sin ^{2} \theta_{i}+\epsilon_{o}} \cdot N_{o} \\
E_{o y} & =\left(-\gamma \sin \theta_{i}+\alpha \sqrt{-\sin ^{2} \theta_{i}+\epsilon_{o}}\right) N_{o} \\
E_{o z} & =\beta \sin \theta_{i} N_{o} \\
N_{o}^{2} & =\frac{1}{\beta^{2} \epsilon_{o}+\left(\gamma \sin \theta_{i}-\alpha \sqrt{-\sin ^{2} \theta_{i}+\epsilon_{o}}\right)^{2}}
\end{aligned}
$$

Also, $q_{e}$ becomes

$$
q_{e}=\frac{\alpha \gamma \sin \theta_{i}\left(\epsilon_{o}-\epsilon_{e}\right)+\sqrt{\epsilon_{o}\left[\gamma^{2} \epsilon_{e}^{2}+\beta^{2} \sin ^{2} \theta_{i}\left(\epsilon_{e}-\epsilon_{o}\right)+\epsilon_{e}\left(\epsilon_{o}-\sin ^{2} \theta_{i}-\gamma^{2} \epsilon_{o}\right)\right]}}{\gamma^{2} \epsilon_{e}+\left(\epsilon_{o}-\gamma^{2} \epsilon_{o}\right)}
$$

and the extraordinary electric field can be written as

$$
\begin{aligned}
E_{e x} & =-\left(\alpha q_{o}^{2}-\gamma q_{e} \sin \theta_{i}\right) / N_{e} \\
E_{e y} & =\beta \epsilon_{o} / N_{e} \\
E_{e z} & =-\left(\gamma-\epsilon_{o}+q_{e}^{2}-\alpha q_{e} \sin \theta_{i}\right) / N_{e} . \\
N_{e}^{2} & =\left(\sin \theta_{i} \gamma q_{e}-\alpha q_{o}^{2}\right)^{2}+\beta^{2} \epsilon_{o}^{2}+\left(\sin \theta_{i} \alpha q_{e}+\gamma q_{e}^{2}-k^{2} \gamma \epsilon_{o}\right)^{2} .
\end{aligned}
$$

$N_{o}$ and $N_{e}$ are normalisation factors that normalise $\mathbf{E}_{o}$ and $\mathbf{E}_{e}$ to unit magnitude.

\subsection{Reflection and Transmission Coefficients}

Now that we are able to express the ordinary and the extraordinary electric field in terms of the optical axis, the refractive indices and the incident ray, it is a simple matter to derive the Fresnel coefficients from the boundary conditions, which imply that the tangential components of $\mathbf{E}$ and $\mathbf{B}$ are continuous:

- If the reflection surface is in the $x y$ plane, $E_{x}$ and $E_{y}$ are continuous.

- The partial derivatives of $E_{x}$ and $E_{y}, \partial E_{x} / \partial z-i K E_{z}$ and $E_{y} / \partial z$, are continuous as well at $z=0$.

From these four boundary conditions we get eight equations. To calculate the Fresnel coefficients, $\mathbf{E}$ has to be decomposed into its two components parallel $(\|)$ and perpendicular $(\perp)$ to the plane of incidence. By decomposing the incoming field into its two different polarised fields, we get four equations for the perpendicular polarised wave [Lekner 1991]

$$
\begin{aligned}
r_{s p} \cos \theta_{i} & =t_{s o} E_{o x}+t_{s e} E_{e x} \\
1+r_{s s} & =t_{s o} E_{o y}+t_{s e} E_{e y} \\
-\cos ^{2} \theta_{i} r_{s p}-\sin ^{2} \theta_{i} r_{s p} & =q_{o} t_{s o} E_{o x}+q_{e} t_{s e} E_{e x}-\sin \theta_{i}\left(t_{s o} E_{o z}+t_{s e} E_{e z}\right) \\
\sin \theta_{i}-\sin \theta_{i} r_{s s} & =q_{o} t_{s o} E_{o y}+q_{e} t_{s e} E_{e y}
\end{aligned}
$$



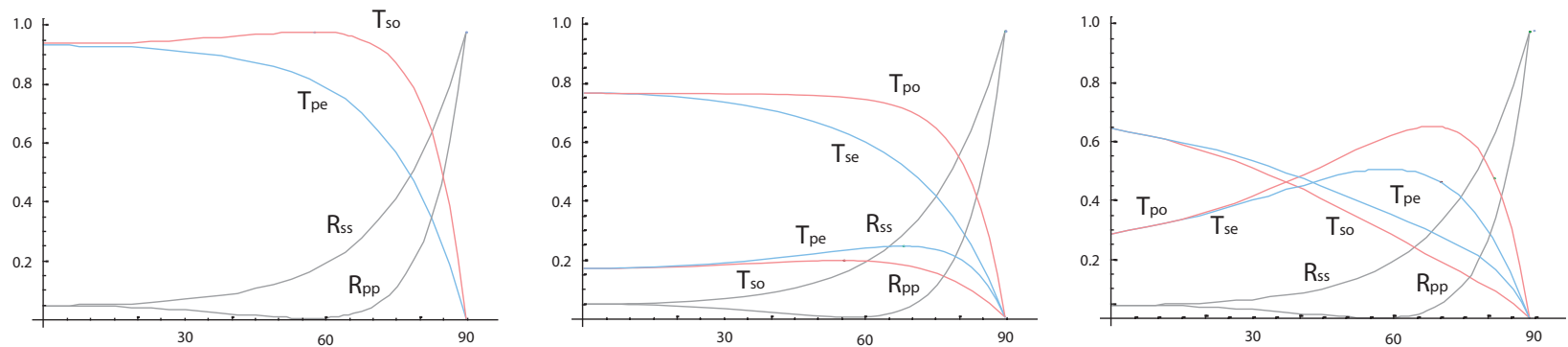

Fig. 3. Fresnel coefficients for uniaxial crystals with $n_{o}=1.54426$ and $n_{e}=1.55335$. Left: Reflection from a basal plane $(\alpha=\beta=0, \gamma=1)$. Middle: Reflection from a plane parallel to the optical axis $(\alpha=\cos \varphi, \beta=\sin \varphi, \gamma=0)$. Right: Arbitrarily oriented optical axis $(\alpha=0.75, \beta=0.5, \gamma=0.433)$.

and four equations for the parallel polarised field.

$$
\begin{aligned}
r_{p s} & =t_{p o} E_{o y}+t_{p e} E_{e y} \\
\cos \theta_{i}+\cos \theta_{i} r_{p p} & =t_{p o} E_{o x}+t_{p e} E_{e x} \\
n_{i} k\left(1-r_{p p}\right) & =q_{o} t_{p o} E_{o x}+q_{e} t_{p e} E_{e x}-\sin \theta_{i}\left(t_{p o} E_{o z}+t_{p e} E_{e z}\right) \\
-\sin \theta_{i} r_{p s} & =q_{o} t_{p o} E_{o y}+q_{e} t_{p e} E_{e y}
\end{aligned}
$$

They have to be solved for the unknown coefficients $r_{s s}, r_{s p}, r_{p p}, r_{p s}, t_{s o}, t_{s e}, t_{p o}$ and $t_{p e}$.

4.3.1 Reflection Amplitudes. $r_{s s}$ and $r_{s p}$ are the reflection amplitudes of an incoming perpendicular polarised wave, $r_{p p}$ and $r_{p s}$ those of an incoming parallel polarised wave. The cross reflection terms $r_{p s}$ and $r_{s p}$ are zero when $\mathbf{E}_{o}$ and $\mathbf{E}_{e}$ are orthogonal, which is not true in general. $r_{p s}=r_{s p}$ when the optical axis lies in the plane of incidence, i.e. if $\gamma=0$, or at normal incidence $(K=0)$.

$$
\begin{aligned}
r_{s s} & =\left[\left(\cos \theta_{i}-q_{e}\right) A E_{e y}-\left(\cos \theta_{i}-q_{o}\right) B E_{o y}\right] / C \\
r_{s p} & =2 n_{i}\left(A E_{e x}-B E_{o x}\right) / C \\
r_{p p} & =\left(2 n_{i}^{2} / \cos \theta_{i} / C\right)\left[\left(\cos \theta_{i}+q_{e}\right) E o x E_{e y}-\left(\cos \theta_{i}+q_{o}\right) E_{e x} E_{o y}\right]-1 \\
r_{p s} & =2 n_{i}\left(q_{e}-q_{o}\right) E_{o y} E_{e y} / C \\
A & =\left(q_{o}+\cos \theta_{i}+\sin \theta_{i} \tan \theta_{i}\right) E_{o x}-\sin \theta_{i} E_{o z} \\
B & =\left(q_{e}+\cos \theta_{i}+\sin \theta_{i} \tan \theta_{i}\right) E_{e x}-\sin \theta_{i} E_{e z} \\
C & =\left(\cos \theta_{i}-q_{e}\right) A E_{e y}-\left(\cos \theta_{i}+q_{o}\right) B E_{o y} .
\end{aligned}
$$

4.3.2 Transmission Amplitudes. $t_{s o}$ and $t_{p o}$ are the transmission amplitudes of the refracted ordinary wave and $t_{s e}$ and $t_{p e}$ those of the extraordinary wave.

$$
\begin{aligned}
t_{s o} & =-2 \cos \theta_{i} B / C \\
t_{p o} & =2 n_{i}\left(\cos \theta_{i}+q_{e}\right) E_{e y} / C \\
t_{s e} & =-2 \cos \theta_{i} A / C \\
r_{p e} & =-2 n_{i}\left(\cos \theta_{i}+q_{o}\right) E_{o y} / C
\end{aligned}
$$

As it can be seen in figure 3, the reflection coefficients hardly change if the direction of the optical axis changes in contrast to the transmission coefficients. If $\left|n_{e}-n_{o}\right|$ is small, the cross reflection terms can be ACM Transactions on Graphics, Vol. TBD, No. TBD, TBD 20TBD. 
neglected, because in that case $r_{p s}$ and $r_{s p}$ are vanishingly small.

The intensity coefficients define the relationship of the radiant intensities of the incoming and the reflected and transmitted waves, respectively

$$
R=\frac{I_{r}}{I_{i}}=r^{2} .
$$

For unpolarised light the reflection coefficient is $R=\left(R_{\perp}+R_{\|}\right) / 2$ and the transmission coefficient $T$ is

$$
T=\frac{I_{t}}{I_{i}}=\frac{n_{t} \cos \theta_{t}}{n_{i} \cos \theta_{i}} t^{2}
$$

$T$ is not $t^{2}$, because the different phase velocities of the two rays and therefore the relation between the refractive indices has to be taken into account. Furthermore $R_{\|}+T_{\|}=1$ and $R_{\perp}+T_{\perp}=1$.

The Fresnel coefficients are not directly applicable to surfaces that are not perfect mirrors (although they may figure as a component in statistical models of rough surfaces), but since crystal surfaces are usually smooth we do not have to concern ourselves with more complex BRDF models here.

\section{DIRECTION COSINE OF THE E-RAY}

\subsection{Previous Work}

Anisotropic materials are included much less frequently in optical systems than isotropic ones; as a result ray tracing of birefringent materials is a rather rare feature even in optical literature.

Nevertheless various methods for ray tracing calculations can still be found. [Simon 1983] started from Maxwell's equations to derive formulas for the direction cosines. Liang's [Liang 1990] method for uniaxial and Zhang's [Zhang 1992] method for biaxial crystals are based on phase matching conditions for wave vectors. [Beyerle and McDermid 1998] proposed analytical expressions for calculating refraction and internal reflection in uniaxial crystals by transforming the coordinate system of the e-ray into a nonorthogonal one so that its normal surface is spherical and follows Snell's law. Furthermore, formulas for the propagation of light can be found in [Swindell 1975], and [McClain et al. 1993b] presented an iterative algorithm for optically active uniaxial crystals.

This calculation of the cosine direction of the extraordinary ray is based on Huygens's principle. According to Huygens's principle every point of a wave front can be seen as centre of a second set of spherical wavelets ${ }^{4}$, so that the wave front can also be considered as the envelope of these wavelets.

\subsection{Derivation of the Direction Cosine}

Starting from the ordinary ray vector $\mathbf{S}_{o}=\left(\xi_{o}, \eta_{o}, \zeta_{o}\right)$, which can be determined by Snell's law, we have to find the ordinary wave front ${ }^{5}$ that passes through the coordinate origin so that

$$
\mathbf{P}_{o} \cdot \mathbf{S}_{o}=x_{o} \xi_{o}+y_{o} \eta_{o}+z_{o} \zeta_{o}=0,
$$

if $\mathbf{P}_{o}=\left(x_{o}, y_{o}, z_{o}\right)$ is the point where the ordinary ray and the ordinary wavelet intersect and $\mathbf{P}_{r}=(h, l, 0)$ is a point on the refracting surface with

$$
\begin{aligned}
x_{o} & =h+\xi_{o} \overline{\mathbf{P}_{r} \mathbf{P}_{o}} / n_{o} \\
y_{o} & =l+\eta_{o} \overline{\mathbf{P}_{r} \mathbf{P}_{o}} / n_{o} \\
x_{o} & =\zeta_{o} \overline{\mathbf{P}_{r} \mathbf{P}_{o}} / n_{o} .
\end{aligned}
$$

\footnotetext{
${ }^{4}$ In this context wavelets are secondary spherical waves originating from each point of the surface.

${ }^{5}$ The ordinary wave front is by definition normal to $\mathbf{S}_{o}$.
} 
The parameter $\overline{\mathbf{P}_{r} \mathbf{P}_{o}}$ is the geometric distance along the ray from $\mathbf{P}_{r}$ to $\mathbf{P}_{o}$. Then the equation for the ordinary wavelet is

$$
\mathcal{F} \equiv\left(\begin{array}{c}
x_{o}-h \\
y_{o}-l
\end{array}\right)^{T}\left(\begin{array}{c}
x_{o}-h \\
y_{o}-l
\end{array}\right)+z_{o}^{2}-n_{o}^{2}\left[\left(\begin{array}{c}
h \\
l
\end{array}\right)^{T}\left(\begin{array}{l}
\xi_{o} \\
\eta_{o}
\end{array}\right)^{2}=0 .\right.
$$

The envelope of the wavelets can be calculated by taking the partial derivatives of $\mathcal{F}$ with respect to $h$ and $l$ and solving the equations for $h$ and $l$.

$$
\left(\begin{array}{c}
h \\
l
\end{array}\right)=\frac{1}{\zeta_{o}^{2}}\left[I-\left(\begin{array}{cc}
\eta_{o}^{2} & -\xi_{o} \eta_{o} \\
-\xi_{o} \eta_{o} & \xi_{o}^{2}
\end{array}\right)\right]\left(\begin{array}{l}
x_{o} \\
y_{o}
\end{array}\right)
$$

This is an expression for the coordinates of the ordinary wavelet centre in terms of the ordinary ray and has to be substituted back into $\mathcal{F}$. $I$ is the identity matrix.

After that, Huygens's extraordinary wavelets have to be found, which are centred on the refracting surface. The ordinary wavelets are replaced by the extraordinary wavelets, where their envelope is the extraordinary wave front, as shown in figure 4. If $\mathbf{A}=(\alpha, \beta, \gamma)$ is the optical axis, the equation of the ellipsoidal wavelets in matrix form is

$$
\mathcal{H} \equiv n_{e}^{2}\left(\begin{array}{c}
x_{e}-h \\
y_{e}-l
\end{array}\right)^{T}+n_{e}^{2} z_{e}^{2}+N\left[\left(\begin{array}{c}
x_{e}-h \\
y_{e}-l
\end{array}\right)^{T}\left(\begin{array}{c}
\alpha \\
\beta
\end{array}\right)+z_{e} \gamma\right]^{2}-n_{o}^{2}\left[\left(\begin{array}{c}
h \\
l
\end{array}\right)^{T}\left(\begin{array}{c}
\xi_{o} \\
\eta_{o}
\end{array}\right)\right]^{2}=0
$$

where

$$
N=n_{o}^{2}-n_{e}^{2}
$$

The extraordinary wavelets have the form of rotationally symmetric ellipsoids which are connected with the ordinary wavelets at the minor axis. The major axis is in the direction of the crystal axis. Again, the envelope of the wavelets can be calculated by taking the partial derivatives of $\mathcal{H}$ with respect to $h$ and $l$. Now the coordinates of the extraordinary wavelet centre in terms of the extraordinary ray are

$$
\begin{array}{r}
\left(\begin{array}{c}
h \\
l
\end{array}\right)=\frac{1}{\Delta^{2}}\left\{\left[n_{o}^{2}\left(n_{e}^{2} I+N\left(\begin{array}{cc}
\beta^{2} & -\alpha \beta \\
-\alpha \beta & \alpha^{2}
\end{array}\right)\right)\left(\begin{array}{cc}
\xi_{o}^{2} & \xi_{o} \eta_{o} \\
\xi_{o} \eta_{o} & \eta_{o}^{2}
\end{array}\right)+\Delta^{2} I\right]\right. \\
\left.\left(\begin{array}{l}
x_{e} \\
y_{e}
\end{array}\right)+N \gamma z_{e}\left[n_{e}^{2} I-n_{o}^{2}\left(\begin{array}{cc}
\eta_{o}^{2} & -\xi_{o} \eta_{o} \\
-\xi_{o} \eta_{o} & \xi_{o}^{2}
\end{array}\right)\right]\left(\begin{array}{l}
\alpha \\
\beta
\end{array}\right)\right\},
\end{array}
$$

with

$$
\Delta^{2}=\Gamma\left[n_{e}^{2}-n_{o}^{2}\left(1-\zeta_{o}^{2}\right)\right]+n_{o}^{2} N \alpha^{2} .
$$

Substituting this back into $\mathcal{H}$ leads to the extraordinary wave-front.

By equating the expressions for the coordinates of the wavelet's origin in terms of the ordinary and the extraordinary rays we eliminate $h$ and $l$ and get the relationship between $\mathbf{P}_{o}$ and $\mathbf{P}_{e}=\left(x_{e}, y_{e}, z_{e}\right)$. $\mathbf{P}_{e}$ is the point where the extraordinary ray and the extraordinary wavelet intersect. Since the extraordinary ray $\mathbf{S}_{e}=\left(\xi_{e}, \eta_{e}, \zeta_{e}\right)$ is a vector from the centre of the wavelet to its point of contact with the extraordinary wave front, i.e. $\mathbf{P}_{e}$, we can find $\mathbf{S}_{e}$ through this.

If we choose a coordinate system $x, y, z$ whose $z$-axis is parallel to the surface normal, the direction cosine of the extraordinary ray is given by the refractive indices $n_{i}, n_{o}$ and $n_{e}$, the direction vector that describe the optical axis $\mathbf{A}$, the direction vector of the ordinary ray and the incident angle $\theta_{i}$.

$$
\left(\begin{array}{l}
\xi_{e} \\
\eta_{e} \\
\zeta_{e}
\end{array}\right)=\frac{1}{\delta_{e g}}\left[\left(\begin{array}{ccc}
n_{o}^{2}\left(n_{e}^{2}+N \beta^{2}\right) & -n_{o}^{2} N \alpha \beta & 0 \\
-n_{o}^{2} N \alpha \beta & n_{o}^{2}\left(n_{e}^{2}+N \alpha^{2}\right) & 0 \\
0 & 0 & \Gamma \Delta / \zeta_{o}
\end{array}\right) \times\left(\begin{array}{l}
\xi_{o} \\
\eta_{o} \\
\zeta_{o}
\end{array}\right)-\gamma N \Delta\left(\begin{array}{lll}
1 & 0 & 0 \\
0 & 1 & 0 \\
0 & 0 & 0
\end{array}\right)\left(\begin{array}{l}
\alpha \\
\beta \\
\gamma
\end{array}\right)\right],
$$

ACM Transactions on Graphics, Vol. TBD, No. TBD, TBD 20TBD. 

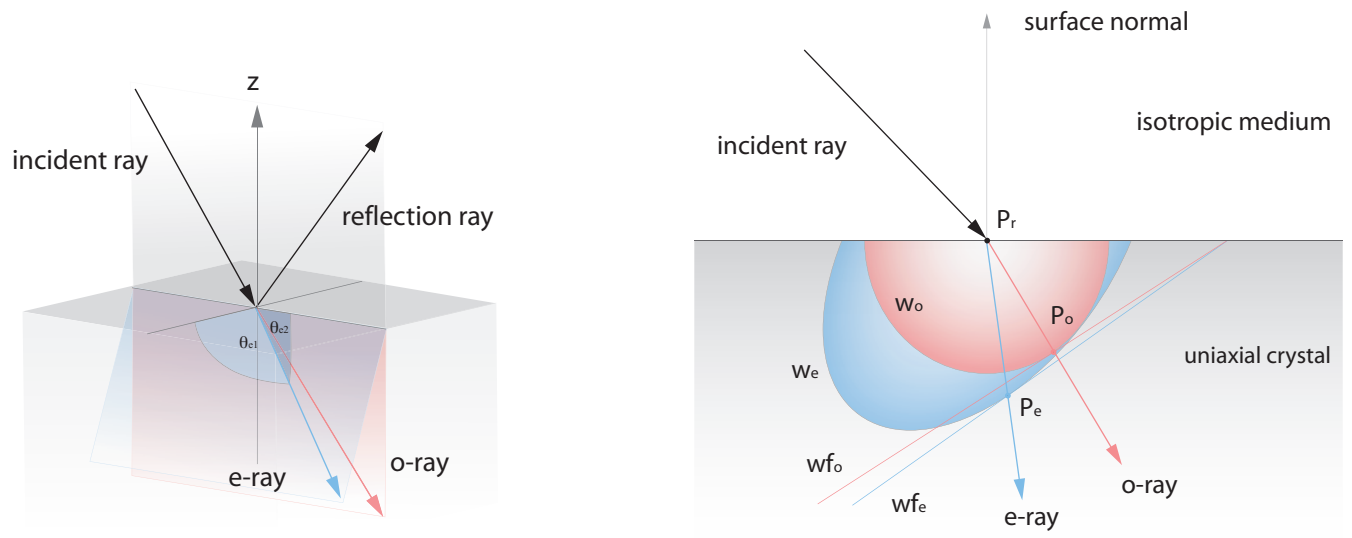

Fig. 4. Left: Incident ray, surface normal, reflective ray and o-ray lie in the plane of incidence. The e-ray does not lie in the plane of incidence. The $z$-axis is in the direction of the surface normal. Right: Huygens's principle; $w_{o}$ and $w_{e}$ are the ordinary and extraordinary wavelets, $w f_{o}$ and $w f_{e}$ are the ordinary and the extraordinary wave fronts.

where

$$
\begin{aligned}
\delta_{e g}^{2} & =n_{e}^{2}\left[n_{o}^{2} \Gamma^{2}-N\left(\gamma \Delta+n_{o}^{2} \alpha\right)^{2}\right], \\
\Gamma & =n_{e}^{2}+N\left(1-\gamma^{2}\right) .
\end{aligned}
$$

The full derivation can be found in [Avendano-Alejo et al. 2002] and [Avendano-Alejo and Stavroudis 2002]. Since we mainly need the direction cosines of the extraordinary ray instead of the ray direction and we can assume the incident refractive index as 1 , the formula reduces to

$$
\theta_{e 1}=\frac{\left(n_{e}^{2}-n_{o}^{2}\right) \alpha\left[n_{o} \beta \sin \theta_{i}+\gamma \cdot G\right]}{\left.\sqrt{n_{e}^{2}\left[n_{e}^{2}\left(n_{e}^{2} n_{o} \gamma^{2}-n_{o}^{3}\left(\gamma^{2}-1\right)\right)^{2}+\left(n_{e}^{2}-n_{o}^{2}\right) \cdot G\right.}\right]^{2}}
$$

and

$$
\theta_{e 2}=\frac{F \cdot G}{\left.\sqrt{n_{e}^{2}\left[n_{e}^{2}\left(n_{e}^{2} n_{o} \gamma^{2}-n_{o}^{3}\left(\gamma^{2}-1\right)\right)^{2}+\left(n_{e}^{2}-n_{o}^{2}\right) \cdot G\right.}\right]^{2}}
$$

with

$$
\begin{aligned}
& F=n_{e}^{2} \gamma^{2}-n_{o}^{2}\left(\gamma^{2}-1\right) \\
& G=\sqrt{F \cdot\left(n_{e}^{2}-1+\cos ^{2} \theta_{i}+\left(n_{o}^{2}-n_{e}^{2}\right) \cdot \beta \sin \theta_{i}\right.}
\end{aligned}
$$

and $\left(\theta_{e 1}, \sqrt{1-\theta_{e 1}^{2}-\theta_{e 2}^{2}}, \theta_{e_{2}}\right)$ after replacing the incident ray with $\left(0, \sin \left(\theta_{i}\right), \cos \left(\theta_{i}\right)\right)$. The numerical results agree with those of [Avendano-Alejo and Stavroudis 2002] and [Beyerle and McDermid 1998].

Generally the surface normal is not aligned with the $z$-axis and a coordinate rotation has to be done to align the equations with the surface normal.

\section{MUELLER MATRICES FOR UNIAXIAL CRYSTALS}

As said before, a characteristic feature of uniaxial crystals is that unpolarised light that passes through it will split into two beams each with a different polarisation. In the following section we will describe these polarisation properties with the help of Stokes vectors and Mueller matrices. 


\subsection{Stokes parameters}

The polarisation state of an electromagnetic wave can be characterized by the Stokes parameters. The four parameters are related to its amplitude and the direction of the orthogonal components of the electric field, $E_{x}$ and $E_{y}$. Only three parameters are necessary to describe the polarisation ellipse, e.g. the amplitudes and the phase difference. The Stokes parameters are defined with $I_{0}$ as the intensity of the light wave and $I_{1}$ as the intensity of the horizontal linear polarisation, $I_{2}$ as the intensity of linear polarisation at an angle of $45^{\circ}$ and $I_{3}$ as intensity of left-handed circularly polarised light [Hecht 1998].

$$
\begin{aligned}
& S_{0}=2 I_{0} \\
& S_{1}=2 I_{1}-2 I_{0} \\
& S_{2}=2 I_{3}-2 I_{0} \\
& S_{3}=2 I_{4}-2 I_{0}
\end{aligned}
$$

For $S_{1}<0$ the light wave tends to be horizontally polarised, if $S_{1}>0$ the polarisation state is vertical rather than horizontal. Otherwise, if $S_{1}=0$ the light wave can be circularly polarised, elliptically polarised at about $\pm 45^{\circ}$ or unpolarised. $S_{2}$ indicates if the light wave is linear polarised at an angle of $+45^{\circ}\left(S_{2}>0\right)$ or $-45^{\circ}\left(S_{2}<0\right)$. Finally, the information about the rotation angle of the wave can be gathered from $S_{3}-$ right $\left(S_{3}>0\right)$, left $\left(S_{3}<0\right)$ or none of them $\left(S_{3}=0\right)$.

The Stokes parameters are related to the electric field parameters by [Hecht 1998]

$$
\begin{aligned}
& S_{0}=\left\langle\mathcal{E}_{x}^{2}\right\rangle+\left\langle\mathcal{E}_{y}^{2}\right\rangle \\
& S_{1}=\left\langle\mathcal{E}_{x}^{2}\right\rangle-\left\langle\mathcal{E}_{y}^{2}\right\rangle \\
& S_{2}=\left\langle 2 \mathcal{E}_{x} \mathcal{E}_{y} \cos \phi\right\rangle \\
& S_{3}=\left\langle 2 \mathcal{E}_{x} \mathcal{E}_{y} \sin \phi\right\rangle .
\end{aligned}
$$

Here, $\phi=\phi_{y}-\phi_{x}$. If light is unpolarised, $\left\langle\mathcal{E}_{x}^{2}\right\rangle=\left\langle\mathcal{E}_{y}^{2}\right\rangle$ and $S_{1}=S_{2}=S_{3}=0$. The Stokes parameters are often normalized by dividing by $S_{0}$. For example the parameters $\left(S_{0}, S_{1}, S_{2}, S_{3}\right)$ for unpolarised light are $(1,0,0,0)$, for horizontally polarised light $(1,1,0,0)$ and for right handed circularly polarised light $(1,0,0,1)$. For completely polarised light

$$
S_{0}^{2}=S_{1}^{2}+S_{2}^{2}+S_{3}^{2}
$$

while for partially polarised light the degree of polarisation is given by

$$
V=\frac{\sqrt{S_{1}^{2}+S_{2}^{2}+S_{3}^{2}}}{S_{0}}
$$

\subsection{Mueller matrices}

While a given polarisation state of light can be completely described by its Stokes vector, the $4 \times 4$ Mueller matrix defines the polarising properties of an optical material which is able to alter the polarisation state of light.

As light passes through an optical device, represented by the Mueller matrix, its polarisation state can change. Multiplying the $4 \times 4$ Mueller matrix $M$ with the input Stokes vector $S_{1}$ of the incident light, the corresponding output Stokes vector $S_{2}$ can be calculated.

$$
S_{2}=M \cdot S_{1}
$$

A device that exhibits more than one property can be represented as a cascade of $N$ optical devices [Azzam and Bashara 1999]

$$
M_{\text {comb }}=M_{N} M_{N-1} \ldots M_{2} M_{1} .
$$

ACM Transactions on Graphics, Vol. TBD, No. TBD, TBD 20TBD. 
Since o- and e-ray are linearly polarised after passing a uniaxial crystal, we do not need to derive the much more complicated Mueller matrices for general retarders or general polarisers. Instead it will suffice to take the Mueller matirx of a linear partial polariser and rotate it to align the input and the output reference coordinate system. The Mueller matrix for $\mathrm{o}^{-}$and e-ray is therefore given by

$$
M_{\text {pol }}=\frac{1}{2}\left(\begin{array}{cccc}
A & B \cos 2 \theta & B \sin 2 \theta & 0 \\
B \cos 2 \theta & A \cos ^{2} 2 \theta+2 C \sin ^{2} 2 \theta & A \cos 2 \theta \cdot \sin 2 \theta-2 C \cos 2 \theta \cdot \sin 2 \theta & 0 \\
B \sin 2 \theta & A \cos 2 \theta \cdot \sin 2 \theta-2 C \cos 2 \theta \cdot \sin 2 \theta & 2 C \cos ^{2} 2 \theta+A \sin ^{2} 2 \theta & 0 \\
0 & 0 & 0 & 2 C
\end{array}\right)
$$

$A=T_{\perp}+T_{\|}, B=T_{\perp}-T_{\|}$and $C=\sqrt{T_{s} T_{p}}$, if $T_{\perp}$ is the transmission coefficient of the o-ray and $T_{\|}$is the transmission coefficient of the e-ray. $\theta$ is measured from the $x$-axis.

This Mueller matrix neglects retardance. The Mueller Matrix for a general phase plate, which produces a phase retardation $\delta$ is

$$
M_{r e t}=\left(\begin{array}{cccc}
1 & 0 & 0 & 0 \\
0 & \cos ^{2} 2 \theta+\sin ^{2} 2 \theta \cdot \beta & \cos 2 \theta \cdot \sin 2 \theta \cdot(1-\beta) & -\sin 2 \theta \cdot \mu \\
0 & \sin 2 \theta \cdot \cos 2 \theta \cdot(1-\beta) & \sin ^{2} 2 \theta+\beta \cdot \cos ^{2} 2 \theta & \cos 2 \theta \cdot \mu \\
0 & \sin 2 \theta \cdot \mu & -\cos 2 \theta \cdot \mu & \beta
\end{array}\right)
$$

with $\beta=\cos \delta$ and $\mu=\sin \delta$. It reduces to the matrices which represent plain glass, half-wave plate and quarter-wave plate when one assumes $\delta$ to be $0, \pi$ and $\pi / 2$, respectively. However, although uniaxial crystals act as retarders and cause a phase shift between $\mathrm{o}^{-}$-and e-ray, we neglect this effect, because the introduced phase difference for a phase plate of thickness $d$

$$
\delta_{e}-\delta_{o}=\frac{2 \pi}{\lambda}\left(n_{e}-n_{o}\right) d
$$

usually cannot be calculated in a ray-based rendering system that relies on standard IEEE floating point arithmetic due to numerical problems, in particular extinction issues.

In order to meaningfully incorporate interference effects in such a system the distances between macroscopic objects along a ray would have to be calculated to a precision of significantly more than the wavelength of light. While IEEE floating point numbers can represent such quantities when taken in isolation, there are not enough significant digits in the mantissa of even the longest float data type to avoid extinction effects after intersection calculations have taken place.

One would need to use an arbitrary precision package such as GNU MP for all these computations, which currently is not feasible due to the enormous slowdown this would mean for the renderer.

\section{INTEGRATION OF BIREFRINGENCE INTO A RAY-BASED RENDERER}

Rendering of uniaxial crystals can be realised through any ray-based algorithm (such as ray or path tracing); the main difference to the isotropic case is that two instead of one transmission rays are sent out on encountering a refraction with a birefringent material. However, some additional alterations are also necessary.

Ordinary and extraordinary rays have different indices of refraction, so two refractive indices have to be stored for each birefringent material as well as the direction cosines of the optical axis. Also, the energy of the incident ray is now distributed differently between the reflection and transmission rays when compared with isotropic materials; the new distribution is governed by the modified Fresnel amplitudes given by equations (3)-(10).

The ordinary reflection ray is calculated as usual, i.e. the direction cosine is determined through Snell's law, and the intensity through the Fresnel coefficients. The procedure is straightforward for the ordinary 
ray, because the index of refraction is constant and independent for each direction.

The calculation of the extraordinary ray is slightly more difficult, because its refractive index depends on the incident angle. Therefore, it has to be computed from scratch for every single extraordinary ray. The usual procedure is to calculate the exact refractive index with the help of the wave normal and to use this solution to calculate the ray vector.

This is rather expensive, so we used the formulas (13) and (14) to compute the ray vector without additional computations. Nonetheless, we need the exact index of refraction for the Fresnel coefficients, but since we have already calculated the direction of the extraordinary ray we only have to build the dot product of the extraordinary ray and the optical axis and evaluate the formula for the refractive index (2). The Fresnel amplitudes are calculated with the formulas (3)-(10). Care should be taken to note that equations (3)-(10) describe the Fresnel amplitudes from which the Fresnel coefficients have to be calculated with the formulas (11) and (12), because the Fresnel coefficients define the relationship of the radiant intensities of the incoming and the reflected and transmitted waves.

\section{RENDERING OF BIREFRINGENCE EFFECTS}

In order to validate our results in practical experiments we implemented the birefringence effects we described as an extension of the standard Fresnel reflection model in the Advanced Rendering Toolkit (ART), the photorealistic rendering system under development at our Institute.

The images were rendered with a standard Whitted ray tracer. Although twice as many transmission rays are sent out for each ray-crystal intersection, we experienced no significant slowdown compared with isotropic materials.

Since these effects are best visible on simple geometries, we used a cube viewed from top on a grid with a luminiscent background instead of actual crystalline structures to demonstrate the various effects which different refractive indices and a change in the direction of the optical axis have on the appearance of crystals in most of our result images. The exception is figure 1, where we used the refractive indices and geometry of calcite to match the appearance of a real crystal specimen.

\subsection{Influence of the Refractive Index}

First we were interested in the consequences of a change of the refractive index on the extraordinary ray. As expected the distance between the doubled lines becomes larger when the difference between $n_{o}$ and $n_{e}-$ and consequently between $\theta_{o}$ and $\theta_{e}$ - was increased as can be seen in figure 5 . Since $n_{o}<n_{e}$, the extraordinary grid lines are below the ordinary grid lines. If $n_{o}>n_{e}$, the doubled lines of the extraordinary ray would be above the original lines. In nature the difference between $n_{o}$ and $n_{e}$ is normally not bigger than 0.3 (for example the refractive indices of rutile are at about 2.616 and 2.903 [Yariv and Yeh 1984]).

\subsection{Influence of the Optical Axis}

We also wanted to show what influence the direction of the optical axis has on the doubling effects. Therefore we rendered four cubes with their optical axes pointing in different direction.

As can be seen in figure 6 , in our setting the double refraction does not occur if the optical axis points in the $z$-direction. This is an understandable result, because when the optical axis points in the $z$-direction, i.e. the viewing direction, all rays that reach the eye travel along the optical axis, and the refractive indices of the ordinary and the extraordinary rays are equal.

The ray surface becomes more and more ellipsoidal from left to right, and the extraordinary ray leaves the plane of incident. Although the direction of the optical axis is decisive for the direction cosines of the extraordinary ray, it influences the Fresnel coefficients and therefore the intensity of the rays only marginally.

Since birefringence depends on the orientation of the object in relation to the optical axis, the doubling effects must change if the object is rotated. To demonstrate this we took a cube with fixed optical axis and 


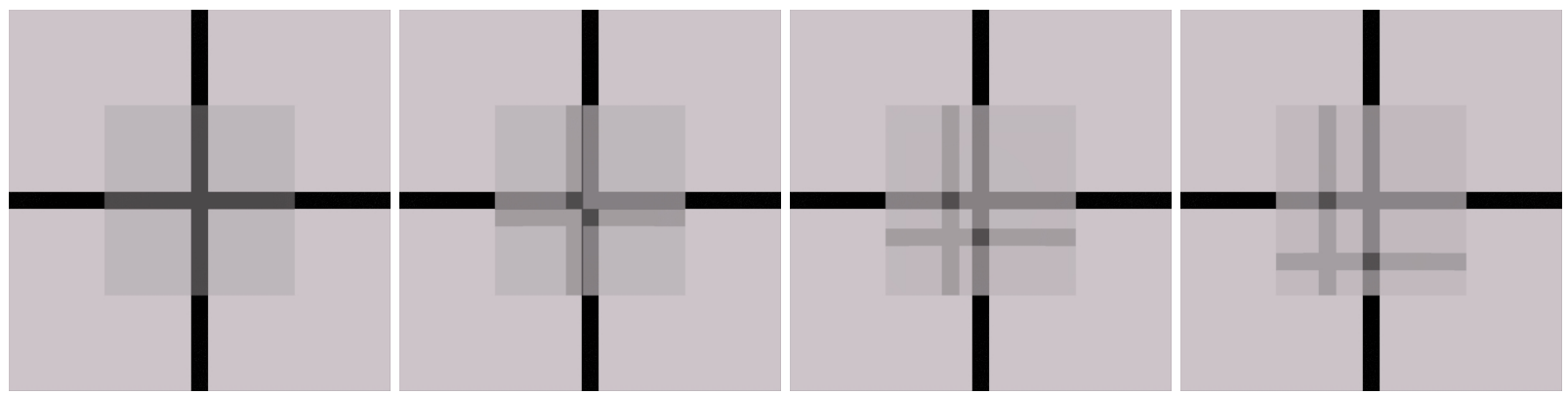

Fig. 5. Cubes with varying indices of refraction. The index of refraction of the extraordinary ray was increased from $n_{e}=1.5$ to $n_{e}=1.6$ to $n_{e}=1.7$ and $n_{e}=1.8$. The refractive index of the ordinary ray is fixed with $n_{o}=1.5$ and the direction of the optical axis is $(-0.577,-0.577,0.577)$. In the beginning, ordinary and extraordinary ray are equal, but with increasing index of refraction the distance between the grid lines becomes larger.
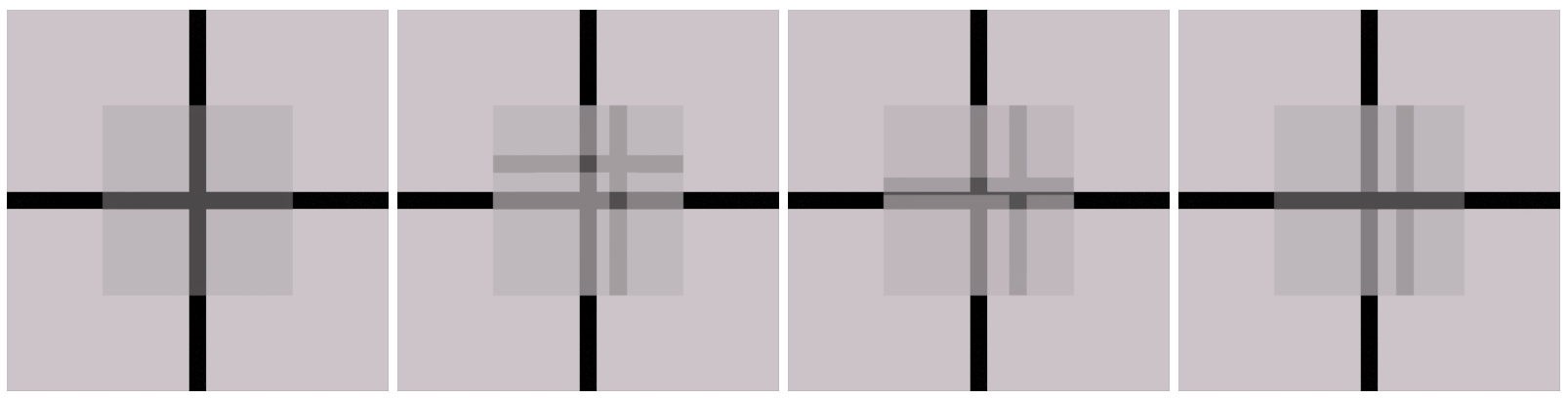

Fig. 6. Cubes with the optical axis in direction - from left to right $-(0,0,1),(0.577,0.577,0.577),(0.904,0.302,0.302)$ and $(0.707,0,0.707)$ and fixed indices of refraction $\left(n_{o}=1.5\right.$ and $\left.n_{e}=1.7\right)$.

refractive indices of $n_{o}=1.35$ and $n_{e}=1.5$. Figure 7 shows the results; when the cube is rotated in steps of $15^{\circ}$, the distance between the horizontal lines becomes larger and larger while the distance of the vertical lines becomes smaller. The effect reaches its maximum at about $45^{\circ}$ and reverses then.

\section{CONCLUSION AND FUTURE WORK}

We have presented a solution for rendering birefringence effects in uniaxial crystals which is based on the current understanding of general light transport in dielectric solids. Using Maxwell's equations and Huygens's principle we have demonstrated how to derive formulas for both the direction cosines of the extraordinary ray and the Fresnel coefficients in a form which is directly applicable to ray tracing calculations.

Our work is limited to the simulation of doubling effects and did not deal with dichroism or absorption within crystals, although these phenomena have to be considered for the rendering of coloured stones. In contrast to birefringence which can be described with the above-mentioned techniques, these effects are direct results of light interacting with the crystal lattice, and - particularly in the case of dichroism - of quantum effects that depend on the type and relative position of the individual atoms in the lattice. Simulation of such phenomena is possible and definitely worthy of future research, but significantly beyond the scope of this paper.

Apart from this it would also be interesting to examine other optical phenomena in crystals like iridescence or to investigate different types of luster; in contrast to dichroism these should be also treatable through 


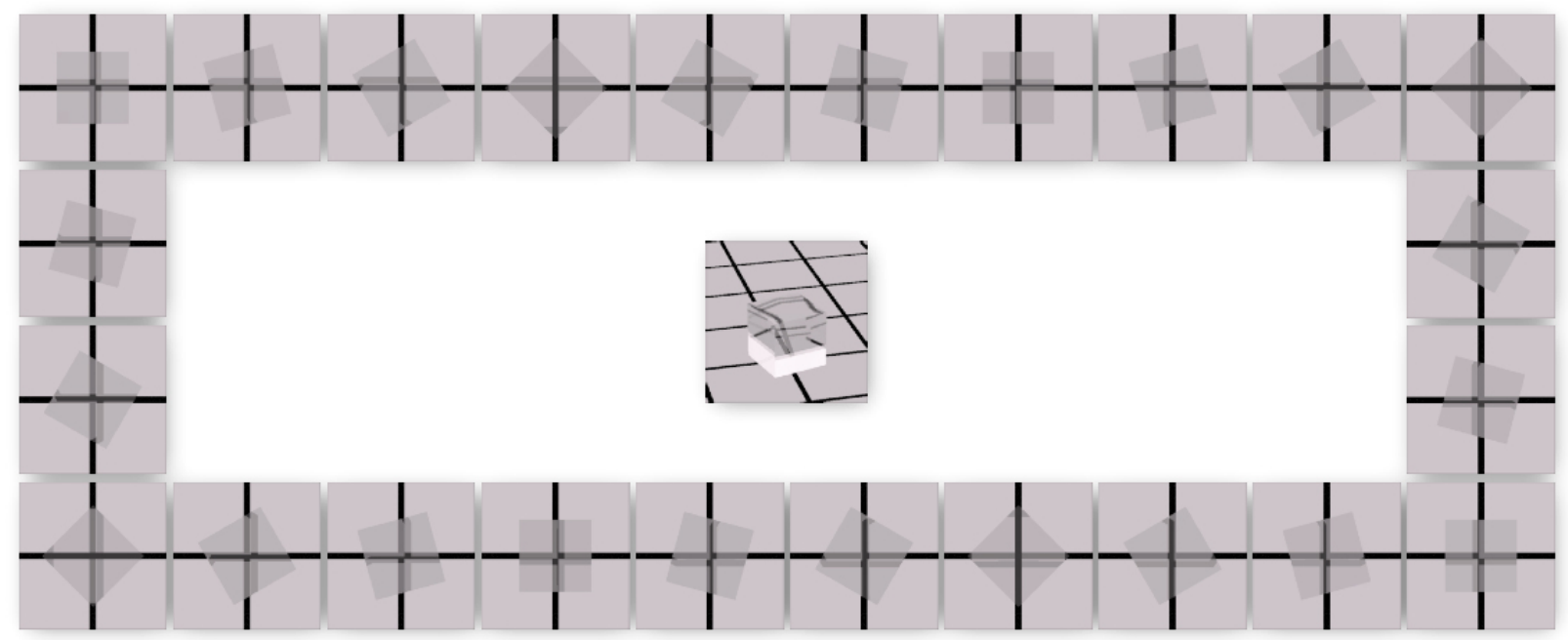

Fig. 7. Orientation-dependency of birefringence. The rotation angle of the cube was increased at steps of $15^{\circ}$ from image to image. $n_{o}=1.3$ and $n_{e}=1.5$. The optical axis was originally $(0.577,0.577,0.577)$.

application of known computer graphics techniques.

Something that would seem to be just a straightforward extension of the uniaxial ray propagation model is the derivation of corresponding formulas for biaxial materials; the derivation of the Fresnel terms for this case would involve changing the coefficients of the dielectric tensor and tracing a second extraordinary ray instead of the ordinary one. However, in this case the mathematical simplifications used for the derivations found in this paper are no longer applicable, and therefore the computation of closed formulas for raycrystal intersections with arbitrary orientation of the optical axes (i.e. the formulas one would need for a ray tracer) is a very complicated problem. The fact that dedicated crystallographic literature contains no general solutions for this problem is a good indicator for this.

Since there are few biaxial crystals which exhibit macroscopically noticeable birefringency effects in the first place - and none of those are commercially used gemstones - we do not plan to investigate this problem further.

\section{REFERENCES}

Avendano-Alejo, M. And Stavroudis, O. N. 2002. Huygens's principle and rays in uniaxial anisotropic media. 2. crystal axis orientation arbitrary. Journal of Optical Society of America 19, 8 (Aug.), 1674-1679.

Avendano-Alejo, M., Stavroudis, O. N., And y Goitia, A. R. B. 2002. Huygens's principle and rays in uniaxial anisotropic media. 1. crystal axis normal to refracting surface. Journal of Optical Society of America 19, 8 (Aug.), 1668-1673.

Azzam, R. M. A. and Bashara, N. M. 1999. Ellipsometry and Polarized Light, 4 ed. Elsevier, Amsterdam.

Beyerle, G. And MCDermid, I. S. 1998. Ray-tracing formulas for refraction and internal reflection in uniaxial crystals. Journal of Optical Society of America 37, 34 (Dec.), 7947-7953.

Born, M. And Wolf, E. 1999. Principles of Optics. Electromagnetic Theory of Propagation, Interference and Diffraction of Light, 7 ed. Cambridge University Press, Cambridge.

Goldstein, H. 2002. Classical Mechanics, 3 ed. Pearson, Upper Saddle River, NJ.

GuY, S. AND Soler, C. 2004. Graphics gems revisited. ACM Transactions on Graphics (Proceedings of the SIGGRAPH conference) 23, 3, 231-238.

Hecht, E. 1998. Optics. 3rd Edition, 3 ed. Addison Wesley Longman, Reading, Mass.

Lekner, J. 1991. Reflection and refraction by uniaxial crystals. Condensed Matter 3, 32 (Aug.), 6121-6133.

ACM Transactions on Graphics, Vol. TBD, No. TBD, TBD 20TBD. 
LiAng, Q.-T. 1990. Simple ray tracing formulas for uniaxial optical crystals. Applied Optics 29, 7 (Mar.), $1008-1010$.

Lin, C.-L. AND Wu, J.-J. 1998. Total reflection of waves propagating from an isotropic medium to an anisotropic medium. Optic Letters 23, 1 (Jan.), 22-24.

McClain, S. C., Hillman, L. W., and Chipman, R. A. 1993a. Polarization ray tracing in anisotropic optically active media. 1. algorithms. Journal of Optical Society of America 10, 11 (Nov.), 2371-2382.

McClain, S. C., Hillman, L. W., And Chipman, R. A. 1993b. Polarization ray tracing in anisotropic optically active media. 2. theory and physics. Journal of Optical Society of America 10, 11 (Nov.), 2383-2393.

Nye, J. F. 2003. Physical Properties of Cystals. Their Representation by Tensors and Matrices. Oxford University Press, Oxford.

Ramachendran, G. N. and Ramaseshan, S. 1961. Crystal optics, diffraction. In Encyclopedia of Physics, S. Flügge, Ed. Vol. 25,1. Julius Springer Verlag, Chapter 1.

Schwelb, O. 1987. Fresnel coefficients for anisotropic media with gain or loss. Journal of Modern Optics 34, 3, 443-453.

Simon, M. C. 1983. Ray tracing formulas for monoaxial optical components. Applied Optics 22, 2 (Jan.), 354-360.

Sun, Y., Fracchia, F., And Drew, M. 2000a. Rendering diamonds. In Proceedings of the 11th Western Computer Graphics Symposium (WCGS). 9-15.

Sun, Y., FracChiA, F. D., AND Drew, M. S. 1999. Rendering the phenomena of volume absorption in homogeneous transparent materials. In 2nd Annual IASTED International Conference on Computer Graphics and Imaging (CGIM '99). 283-288.

Sun, Y., Fracchia, F. D., And Drew, M. S. 2000b. Rendering light dispersion with a composite spectral model. In 1st International Conference on Color in Graphics and Image Processing. 51-56.

Swindell, W. 1975. Extraordinary-ray and -wave tracing in uniaxial crystals. Applied Optics 14, 9 (Sept.), $2298-2301$.

SzIVessy, G. 1928. Licht als Wellenbewegung. In Handbuch der Physik, H. Geiger and K. Scheel, Eds. Vol. 20. Julius Springer Verlag, Berlin, Chapter 11.

Tannenbaum, D. C., Tannenbaum, P., And Wozny, M. J. 1994. Polarization and birefringency considerations in rendering. In Proceedings of SIGGRAPH '94 (Orlando, Florida, July 24-29, 1994), A. Glassner, Ed. Computer Graphics Proceedings, Annual Conference Series. ACM SIGGRAPH, ACM Press, 221-222. ISBN 0-89791-667-0.

Thomas, S. W. 1986. Dispersive refraction in ray tracing. The Visual Computer 2, 1 (Jan.), 3-8.

Wilkie, A., Tobler, R. F., And Purgathofer, W. 2001. Combined rendering of polarization and fluorescence effects. In Proceedings of the 12th Eurographics Workshop on Rendering Techniques. Springer-Verlag, 197-204.

Wolff, L. B. And Kurlander, D. 1990. Ray tracing with polarization parameters. IEEE Computer Graphics ES Applications 10, 6 (Nov.), 44-55.

Yariv, A. And Yeh, P. 1984. Optical Waves in Crystals. Propagation and control of Laser Radiation. Wiley-Interscience Publication, New York.

Yokoi, S., Kurashige, K., And ichiro Toriwaki, J. 1986. Rendering gems with asterism or chatoyancy. The Visual Computer 2, 5, 307-312.

Yuan, Y., Kunit, T. L., Inamoto, N., And Sun, L. 1988. Gemstone fire: adaptive dispersive ray tracing of polyhedrons. The Visual Computer 4, 5, 259-270.

Zhang, W.-Q. 1992. General ray-tracing formulas for crystals. Applied Optics 31, 34 (Dec.), 7328-7331. 